\title{
Some comments on the integrability of the noncommutative generalized massive Thirring model
}

\author{
H. Blas *广 \\ Instituto de Física - Universidade Federal de Mato Grosso \\ Cuiabá-Brazil \\ E-mail: blasefisica.ufmt.br \\ H. L. Carrion \\ Instituto de Física, Universidade de São Paulo, \\ Caixa Postal 68528, 21941-972 São Paulo, Brazil \\ E-mail: hectorseect.ufrn.br

\section{B. M. Cerna} \\ Departamento de Ciencias, Escuela de Matemáticas \\ Universidad Nacional Santiago Antunez de Mayolo \\ Huaraz-Perú \\ E-mail: bcernameunasam.edu.pe
}

\begin{abstract}
Some properties of a non-commutative version of the generalized massive Thirring theory (NCGMT) are studied. We develop explicit calculations for the affine Lie algebra $g l(3)$ case. The NCGMT model is written in terms of Dirac type fields corresponding to the Moyal product extension of the ordinary multi-field massive Thirring model. We discuss the Lagrangian formulation, its zero-curvature representation and integrability property of certain submodels.
\end{abstract}

5th International School on Field Theory and Gravitation,

April 20 - 242009

Cuiabá city, Brazil

\footnotetext{
*Poster Section

${ }^{\dagger} \mathrm{HB}$ thanks CNPq for partial support

†Permanent address: Escola de Ciências e Tecnologia / ECT-UFRN Av. Hermes da Fonseca, 1111, Tirol 59014-615 Natal-RN. HLC thanks FAPESP for support during the initial stage of the work
} 
The massive Thirring (MT) model and its dual the sine-Gordon model are known to be integrable. Some versions of their multi-field extensions have appeared in the literature (see e.g. [1]). On the other hand, the noncommutative (NC) version of the MT model has been proposed recently [2]. Here we discuss some properties of the affine Kac-Moody algebraic formulation of a noncommutative version of the so-called generalized massive Thirring model.

\section{A version of the noncommutative generalized massive Thirring model (NCGMT)}

The ref. [2] presents a version of the so-called NC massive Thirring model. Here we address the non-commutative version of the generalized model presented in [1]. It is a two-dimensional spinor model in which the usual Thirring self-interaction term for each species is extended with similar interactions among different massive spinor field species. The action for the NCGMT model in terms of the massive Thirring field components becomes

$$
\begin{aligned}
S_{N C M T} & =\int d x^{2} \sum_{i=1}^{i=3}\left\{\left[2 i \widetilde{\psi}_{L}^{i} \star \partial_{+} \psi_{L}^{i}+2 i \widetilde{\psi}_{R}^{i} \star \partial_{-} \psi_{R}^{i}+i m_{i}\left(\widetilde{\psi}_{L}^{i} \star \psi_{R}^{i}-\psi_{L}^{i} \star \widetilde{\psi}_{R}^{i}\right)\right]\right. \\
& \left.-2\left(A_{L}^{i} \star A_{R}^{i}\right)\right\},
\end{aligned}
$$

the star $\star$ represents the Moyal product and

$$
\begin{aligned}
& A_{R}^{1}=\sqrt[4]{\frac{\alpha_{1} \beta_{1}}{4}} \psi_{R}^{1} \star \tilde{\psi}_{R}^{1}+\sqrt[4]{\frac{\beta_{3} \alpha_{3}}{4}} \psi_{R}^{3} \star \tilde{\psi}_{R}^{3} \\
& A_{R}^{2}=\sqrt[4]{\frac{\alpha_{2} \beta_{2}}{4}} \psi_{R}^{2} \star \tilde{\psi}_{R}^{2}-\sqrt[4]{\frac{\alpha_{1} \beta_{1}}{4}} \tilde{\psi}_{R}^{1} \star \psi_{R}^{1} \\
& A_{R}^{3}=\sqrt[4]{\frac{\beta_{3} \alpha_{3}}{4}} \tilde{\psi}_{R}^{3} \star \psi_{R}^{3}+\sqrt[4]{\frac{\alpha_{2} \beta_{2}}{4}} \tilde{\psi}_{R}^{2} \star \psi_{R}^{2} \\
& A_{L}^{1}=\sqrt[4]{\frac{\delta_{1} \lambda_{1}}{4}} \psi_{L}^{1} \star \tilde{\psi}_{L}^{1}+\sqrt[4]{\frac{\delta_{3} \lambda_{3}}{4}} \psi_{L}^{3} \star \tilde{\psi}_{L}^{3} \\
& A_{L}^{2}=\sqrt[4]{\frac{\delta_{2} \lambda_{2}}{4}} \psi_{L}^{2} \star \tilde{\psi}_{L}^{2}-\sqrt[4]{\frac{\delta_{1} \lambda_{1}}{4}} \tilde{\psi}_{L}^{1} \star \psi_{L}^{1} \\
& A_{L}^{3}=\sqrt[4]{\frac{\delta_{3} \lambda_{3}}{4}} \tilde{\psi}_{L}^{3} \star \psi_{L}^{3}+\sqrt[4]{\frac{\delta_{2} \lambda_{2}}{4}} \tilde{\psi}_{L}^{2} \star \psi_{L}^{2}
\end{aligned}
$$

Now we write the equations of motion

$$
\begin{aligned}
& \partial_{+} \psi_{L}^{3}=-\frac{1}{2} m_{3} \psi_{R}^{3}-i \sqrt[4]{\frac{\delta_{3} \lambda_{3}}{4}}\left\{\psi_{L}^{3} \star A_{R}^{3}+A_{R}^{1} \star \psi_{L}^{3}\right\} \\
& \partial_{+} \tilde{\psi}_{L}^{1}=-\frac{1}{2} m_{1} \tilde{\psi}_{R}^{1}+i \sqrt[4]{\frac{\delta_{1} \lambda_{1}}{4}}\left\{\tilde{\psi}_{L}^{1} \star A_{R}^{1}-A_{R}^{2} \star \tilde{\psi}_{L}^{1}\right\} \\
& \partial_{+} \tilde{\psi}_{L}^{2}=-\frac{1}{2} m_{2} \tilde{\psi}_{R}^{2}+i \sqrt[4]{\frac{\delta_{2} \lambda_{2}}{4}}\left\{\tilde{\psi}_{L}^{2} \star A_{R}^{2}+A_{R}^{3} \star \tilde{\psi}_{L}^{2}\right\} . \\
& \partial_{+} \tilde{\psi}_{L}^{3}=-\frac{1}{2} m_{3} \tilde{\psi}_{R}^{3}+i \sqrt[4]{\frac{\delta_{3} \lambda_{3}}{4}}\left\{A_{R}^{3} \star \tilde{\psi}_{L}^{3}+\tilde{\psi}_{L}^{3} \star A_{R}^{1}\right\} \\
& \partial_{+} \psi_{L}^{1}=-\frac{1}{2} m_{1} \psi_{R}^{1}-i \sqrt[4]{\frac{\delta_{1} \lambda_{1}}{4}}\left\{A_{R}^{1} \star \psi_{L}^{1}-\psi_{L}^{1} \star A_{R}^{2}\right\}
\end{aligned}
$$




$$
\begin{aligned}
& \partial_{+} \psi_{L}^{2}=-\frac{1}{2} m_{2} \psi_{R}^{2}-i \sqrt[4]{\frac{\delta_{2} \lambda_{2}}{4}}\left\{A_{R}^{2} \star \psi_{L}^{2}+\psi_{L}^{2} \star A_{R}^{3}\right\} . \\
& \partial_{-} \psi_{R}^{3}=\frac{1}{2} m_{3} \psi_{L}^{3}-i \sqrt[4]{\frac{\alpha_{3} \beta_{3}}{4}}\left\{\psi_{R}^{3} \star A_{L}^{3}+A_{L}^{1} \star \psi_{R}^{3}\right\} \\
& \partial_{-} \tilde{\psi}_{R}^{1}=\frac{1}{2} m_{1} \tilde{\psi}_{L}^{1}+i \sqrt[4]{\frac{\alpha_{1} \beta_{1}}{4}}\left\{\tilde{\psi}_{R}^{1} \star A_{L}^{1}-A_{L}^{2} \star \tilde{\psi}_{R}^{1}\right\} \\
& \partial_{-} \tilde{\psi}_{R}^{2}=\frac{1}{2} m_{2} \tilde{\psi}_{L}^{2}+i \sqrt[4]{\frac{\alpha_{2} \beta_{2}}{4}}\left\{\tilde{\psi}_{R}^{2} \star A_{L}^{2}+A_{L}^{3} \star \tilde{\psi}_{R}^{2}\right\} . \\
& \partial_{-} \tilde{\psi}_{R}^{3}=\frac{1}{2} m_{3} \tilde{\psi}_{L}^{3}+i \sqrt[4]{\frac{\alpha_{3} \beta_{3}}{4}}\left\{A_{L}^{3} \star \tilde{\psi}_{R}^{3}+\tilde{\psi}_{R}^{3} \star A_{L}^{1}\right\} \\
& \partial_{-} \psi_{R}^{1}=\frac{1}{2} m_{1} \psi_{L}^{1}-i \sqrt[4]{\frac{\alpha_{1} \beta_{1}}{4}}\left\{A_{L}^{1} \star \psi_{R}^{1}-\psi_{R}^{1} \star A_{L}^{2}\right\} \\
& \partial_{-} \psi_{R}^{2}=\frac{1}{2} m_{2} \psi_{L}^{2}-i \sqrt[4]{\frac{\alpha_{2} \beta_{2}}{4}}\left\{A_{L}^{2} \star \psi_{R}^{2}+\psi_{R}^{2} \star A_{L}^{3}\right\} .
\end{aligned}
$$

The set of equations of motion above are the $g l(3)$ extension of the equations of motion given before for the case $g l(2) \mathrm{NCMT}_{1}$ ( see eqs. (5.11)-(5.14) of ref. [2]). In fact, the later system is contained in the $g l(3)$ extended model. For example, if one considers $\psi_{L}^{1}=\psi_{L}^{2}=\tilde{\psi}_{L}^{1}=\tilde{\psi}_{L}^{1}=0$ in the eq. (1.11) then it is reproduced the equation (5.13) of reference [2] describing the single Thirring field $\psi_{3}$ provided that the parameters expression $\sqrt[4]{\frac{\delta_{3} \lambda_{3} \beta_{3} \alpha_{3}}{16}}$ corresponds to the coupling constant $\frac{\lambda}{2}$ of NCMT model as defined in that reference.

The four field interaction terms in the action (1.1) can be re-written as a sum of Dirac type current-current terms for the various flavors $(j=1,2,3)$. In the constructions of the relevant currents the double-gauging of a $U(1)$ symmetry in the star-localized Noether procedure deserve a careful treatment [2]. So, one has two types of currents for each flavor [2]

$$
\begin{aligned}
j_{k}^{(1) \mu} & =\bar{\psi}_{k} \gamma^{\mu} \star \psi_{k}, \\
j_{k}^{(2) \mu} & =-\psi_{k}^{T} \gamma^{0} \gamma^{\mu} \star \widetilde{\psi}_{k}, \quad k=1,2,3 . .
\end{aligned}
$$

In order to write as a sum of current-current interaction terms it is necessary to impose the next constraints on the coupling parameters $\frac{\delta_{j} \lambda_{j}}{\alpha_{j} \beta_{j}}=\kappa=$ const.; $j=1,2,3$. with $\kappa^{3}=1$. Then the current-current terms can be written as

$$
\begin{aligned}
-2 \sum_{i=1}^{3} A_{L}^{i} \star A_{R}^{i}= & -g_{11}\left(j_{1 \mu}^{(1)} \star j_{1}^{(1) \mu}+j_{1 \mu}^{(2)} \star j_{1}^{(2) \mu}\right)-g_{22}\left(j_{2 \mu}^{(1)} \star j_{2}^{(1) \mu}+j_{2 \mu}^{(2)} \star j_{2}^{(2) \mu}\right)- \\
& g_{33}\left(j_{3 \mu}^{(1} \star j_{3}^{(1) \mu}+j_{3 \mu}^{(2)} \star j_{3}^{(2) \mu}\right)+g_{12}\left(j_{1 \mu}^{(1)} \star j_{2}^{(2) \mu}\right)- \\
& g_{23}\left(j_{2 \mu}^{(1)} \star j_{3}^{(1) \mu}\right)-g_{13}\left(j_{1 \mu}^{(2)} \star j_{3}^{(2) \mu}\right),
\end{aligned}
$$

where

$$
g_{j j}=\frac{1}{4} \sqrt[4]{\alpha_{j} \beta_{j} \delta_{j} \lambda_{j}}, \quad g_{j k}=\frac{1}{2} \sqrt[4]{\alpha_{j} \beta_{j} \delta_{k} \lambda_{k}},(j \neq k) ; \quad j, k=1,2,3 .
$$

The two type of $\mathrm{U}(1)$ currents $j_{k \mu}^{(1)}, j_{k \mu}^{(2)}(\mathrm{k}=1,2,3)$, respectively, satisfy the equations

$$
\partial_{+}\left(\tilde{\psi}_{L}^{k} \star \psi_{L}^{k}\right)+\partial_{-}\left(\tilde{\psi}_{R}^{k} \star \psi_{R}^{k}\right)=0, \quad \partial_{+}\left(\psi_{L}^{k} \star \tilde{\psi}_{L}^{k}\right)+\partial_{-}\left(\psi_{R}^{k} \star \tilde{\psi}_{R}^{k}\right)=0, \quad k=1,2,3 .
$$




\section{Matrix valued fields in the action and the zero curvature condition}

We propose the NCGMT action related to the fields $W_{m}^{ \pm}$as

$$
\begin{aligned}
S\left[W_{m}^{ \pm}\right]= & \sum_{m=1}^{2} \int d x^{2}\left\{\frac{1}{2}<\left[E_{-3}, W_{3-m}^{+}\right] \star \partial_{+} W_{m}^{+}>-\frac{1}{2}<\left[E_{3}, W_{3-m}^{-}\right] \star \partial_{-} W_{m}^{-}>-\right. \\
& \left.<\left[E_{-3}, W_{m}^{+}\right] \star\left[E_{3}, W_{m}^{-}\right]>\right\}-\frac{1}{2} \sum_{m, n=1}^{2}<\hat{J}_{m}^{+} \star \hat{J}_{n}^{-}>.
\end{aligned}
$$

The action above possesses some global symmetries and the associated matrix-valued currents

$$
J_{m}^{ \pm}= \pm \frac{1}{4}\left[\left[E_{\mp 3}, W_{m}^{ \pm}\right], W_{3-m}^{ \pm}\right]_{\star}
$$

Notice that the currents $J_{m}^{ \pm}$, despite the indices in their notation, has zero gradation. The fields $W_{m}^{ \pm}(m=1,2)$ and the matrix element $E_{ \pm 3}$ are given in the appendix A, and they have gradation $\pm m$ and \pm 3 , respectively. The hatted fields mean that the spinor fields have been re-scaled conveniently by $\hat{W}_{m}^{ \pm}=L_{m}^{ \pm} W_{m}^{ \pm}\left(L_{m}^{ \pm}\right)^{-1}$, where $L_{m}^{ \pm}$are some constant matrices.

The zero-curvature condition encodes integrability even in the NC extension of integrable models (see e.g. [2] and references therein), as this condition allows, for example, the construction of infinite conserved charges for them. The $g l(3)$ NCGMT model equations of motion (1.8)-(1.19) can be formulated as a zero curvature condition by considering the following Lax pair

$$
\begin{gathered}
A_{-}=E_{-3}+a\left[E_{-3}, W_{1}^{+}\right]_{\star}+b\left[E_{-3}, W_{2}^{+}\right]_{\star}+g_{1}\left[\left[E_{-3}, \hat{W}_{1}^{+}\right], \hat{W}_{2}^{+}\right]_{\star}+g_{2}\left[\left[E_{-3}, \hat{W}_{2}^{+}\right], \hat{W}_{1}^{+}\right]_{\star} \\
A_{+}=-E_{+3}+b\left[E_{+3}, W_{1}^{-}\right]_{\star}+a\left[E_{+3}, W_{2}^{-}\right]_{\star}++\widetilde{g}_{1}\left[\left[E_{+3}, \hat{W}_{1}^{-}\right], \hat{W}_{2}^{-}\right]_{\star}+\widetilde{g}_{2}\left[\left[E_{+3}, \hat{W}_{2}^{-}\right], \hat{W}_{1}^{-}\right]_{\star},
\end{gathered}
$$

where $a, b, g_{1}, g_{2}, \widetilde{g}_{1}, \widetilde{g}_{2}$ are some parameters to be determined below. These matrix valued fields must be replaced into the zero-curvature equation

$$
\left[\partial_{+}+A_{+}, \partial_{-}+A_{-}\right]_{\star}=0
$$

In order to get the relevant equations of motion it is useful to take into consideration the gradation structure of the various terms. So, we can expand explicitly the terms of gradation $(-1)$ obtaining

$$
\left[E_{-3}, \partial_{+} W_{2}^{+}\right]_{\star}=+\left[E_{-3},\left[E_{3}, W_{1}^{-}\right]\right]_{\star}-\left(4 g_{1}+4 g_{2}\right)\left(L_{2}^{+}\right)^{-1}\left[\hat{J}_{1}^{-},\left[E_{-3}, \hat{W}_{2}^{+}\right]\right]_{\star} L_{2}^{+}
$$

plus the constraint

$$
\left[F_{1}^{+}, F_{2}^{-}\right]_{\star}=0
$$

Next, looking for the gradation +1 terms we arrive at the equation

$$
\left[E_{3}, \partial_{-} W_{2}^{-}\right]_{\star}=-\left[E_{3},\left[E_{-3}, W_{1}^{+}\right]\right]_{\star}-(4 g 1+4 g 2)\left(L_{2}^{-}\right)^{-1}\left[\hat{J}_{1}^{+},\left[E_{3}, \hat{W}_{2}^{-}\right]\right]_{\star} L_{2}^{-},
$$

plus the constraint

$$
\left[F_{2}^{+}, F_{1}^{-}\right]_{\star}=0
$$


Following the process we can write similar eqs. for the \pm 2 gradations. We conclude that in order to obtain the equations of motion (1.8)-(1.19) it is required the conditions $L_{2}^{ \pm}=L_{1}^{ \pm}$and $g_{1}=g_{2}=-\frac{1}{4}$, provided that the constraints (2.5) and (2.7) are imposed.

Finally, for the zero gradation term there appears the following equation

$$
\begin{array}{r}
\partial_{+} \hat{J}_{1}^{+}+\partial_{+} \hat{J}_{2}^{+}-\partial_{-} \hat{J}_{1}^{-}+\partial_{-} \hat{J}_{2}^{-}-a b\left[F_{2}^{+}, F_{2}^{-}\right]-a b\left[F_{1}^{+}, F_{1}^{-}\right]+\left[\hat{J}_{1}^{-}, \hat{J}_{1}^{+}\right]+\left[\hat{J}_{1}^{-}, \hat{J}_{2}^{+}\right]+ \\
{\left[\hat{J}_{2}^{-}, \hat{J}_{1}^{+}\right]+\left[\hat{J}_{2}^{-}, \hat{J}_{2}^{+}\right]=0 .}
\end{array}
$$

Taking into account the conditions $\hat{J}_{1}^{+}=\hat{J}_{2}^{+}$and $\hat{J}_{1}^{-}=\hat{J}_{2}^{-}$in the above equation we may write it as

$$
\partial_{+} \hat{J}_{1}^{+}-\partial_{-} \hat{J}_{1}^{-}-\frac{a b}{2}\left[F_{2}^{+}, F_{2}^{-}\right]-\frac{a b}{2}\left[F_{1}^{+}, F_{1}^{-}\right]+2\left[\hat{J}_{1}^{-}, \hat{J}_{1}^{+}\right]=0 .
$$

The zero gradation equation must be consistent with the equations of motion described above. In order to see the form of these three equations let us write one of the them in terms of the fundamental fields

$$
\begin{aligned}
i\left(\partial_{+} A_{L}^{3}-\partial_{-} A_{R}^{3}\right)_{\star}= & \left(A_{R}^{3} \star A_{L}^{3}-A_{L}^{3} \star A_{R}^{3}\right)-a b\left\{i m_{3}\left(\sqrt[4]{\frac{\beta_{3} \lambda_{3}}{4}} \tilde{\psi}_{R}^{3} \star \psi_{L}^{3}+\sqrt[4]{\frac{\delta_{3} \alpha_{3}}{4}} \tilde{\psi}_{L}^{3} \star \psi_{R}^{3}\right)+\right. \\
& \left.\operatorname{im}_{2}\left(\sqrt[4]{\frac{\delta_{2} \alpha_{2}}{4}} \tilde{\psi}_{R}^{2} \star \psi_{L}^{2}+\sqrt[4]{\frac{\beta_{2} \lambda_{2}}{4}} \tilde{\psi}_{L}^{2} \star \psi_{R}^{2}\right)\right\}
\end{aligned}
$$

In particular, if we reduce the eq. (2.10) to get an equation for just one field, say $\psi^{3}$, one has

$$
\begin{aligned}
-\sqrt[4]{\frac{\delta_{3} \lambda_{3}}{4}} \partial_{+}\left(\tilde{\psi}_{L}^{3} \star \psi_{L}^{3}\right)+\sqrt[4]{\frac{\beta_{3} \alpha_{3}}{4}} \partial_{-}\left(\tilde{\psi}_{R}^{3} \star \psi_{R}^{3}\right)= & a b m_{3}\left(\sqrt[4]{\frac{\beta_{3} \lambda_{3}}{4}} \tilde{\psi}_{R}^{3} \star \psi_{L}^{3}+\sqrt[4]{\frac{\delta_{3} \alpha_{3}}{4}} \tilde{\psi}_{L}^{3} \star \psi_{R}^{3}\right)+ \\
& i \sqrt[4]{\frac{\delta_{3} \alpha_{3} \beta_{3} \lambda_{3}}{16}}\left(\tilde{\psi}_{R}^{3} \star \psi_{R}^{3} \star \tilde{\psi}_{L}^{3} \star \psi_{L}^{3}-\tilde{\psi}_{L}^{3} \star \psi_{L}^{3} \star \tilde{\psi}_{R}^{3} \star \psi_{R}^{3}\right)
\end{aligned}
$$

Now, taking into account the relationships $\frac{\delta_{j} \lambda_{j}}{\alpha_{j} \beta_{j}}=1 ; j=1,2,3$ with the condition $\lambda_{3} \beta_{3}=\delta_{3} \alpha_{3}$ and the identifications $\sqrt[4]{\frac{\lambda_{3} \delta_{3}}{4}} \rightarrow-\lambda, a b m_{3} \rightarrow m_{\psi}$ we arrive at the equation (5.18) of the ref. [2] .

In terms of the fundamental fields the constraints (2.5) and (2.7) have the following form

$$
\psi_{R}^{1} * \psi_{L}^{2}=\psi_{L}^{1} * \psi_{R}^{2}, \quad \psi_{R}^{2} * \tilde{\psi}_{L}^{3}=-\psi_{L}^{2} * \tilde{\psi}_{R}^{3}, \quad \tilde{\psi}_{L}^{3} * \psi_{R}^{1}=-\tilde{\psi}_{R}^{3} * \psi_{L}^{1}
$$

and

$$
\psi_{R}^{3} * \tilde{\psi}_{L}^{2}=-\psi_{L}^{3} * \tilde{\psi}_{R}^{2}, \quad \tilde{\psi}_{L}^{1} * \psi_{R}^{3}=-\tilde{\psi}_{R}^{1} * \psi_{L}^{3}, \quad \tilde{\psi}_{R}^{2} * \tilde{\psi}_{L}^{1}=\tilde{\psi}_{L}^{2} * \tilde{\psi}_{R}^{1},
$$

respectively.

The action (1.1) (or its matrix form (2.1)) defines a three species NC generalized massive Thirring model since one has six complex fields, i.e. $\psi_{R, L}^{j}$ and its complex conjugates $\widetilde{\psi}_{R, L}^{j},(j=$ $1,2,3)$. The zero-curvature formulation requires the above six constraints (2.12) and (2.13). This fact suggests that the NCGMT model defined by the action (1.1) becomes integrable only for a submodel defined by the eqs. of motion (1.8)-(1.19) provided the constraints (2.5) and (2.7) are satisfied. So, one expects that a careful introduction of the constraints trough relevant Lagrange 
multipliers into the action will provide the lagrangian formulation of an integrable submodel of the NCGMT theory.

Regarding the action related to the zero curvature equations without constraints it is interesting to notice that the quadratic terms in the equations of motion make it difficult to believe that one can find a local lagrangian for the theory. Obviously, in that case we could not have a generalized massive Thirring model with a local lagrangian involving bilinear (kinetic and mass terms) and current-current terms.

\section{A. The sl(3) affine Kac-Moody algebra and the matrix fields}

The matrix fields entering the potentials take the form

$$
\begin{aligned}
F_{m}^{ \pm} & =\mp\left[E_{ \pm 3}, W_{3-m}^{\mp}\right], m=1,2 \\
E_{ \pm 3} & =\frac{1}{6}\left[\left(2 m_{1}+m_{2}\right) H_{1}^{ \pm 1}+\left(2 m_{2}+m_{1}\right) H_{2}^{ \pm 1}\right], \quad m_{3}=m_{1}+m_{2} \\
W_{1}^{-} & =-\sqrt{\frac{4 i}{m_{3}}} \psi_{R}^{3} E_{\alpha 3}^{-1}+\sqrt{\frac{4 i}{m_{1}}} \widetilde{\psi}_{R}^{1} E_{-\alpha 1}^{0}+\sqrt{\frac{4 i}{m_{2}}} \widetilde{\psi}_{R}^{2} E_{-\alpha 2}^{0} \\
W_{1}^{+} & =\sqrt{\frac{4 i}{m_{1}}} \psi_{L}^{1} E_{\alpha 1}^{0}+\sqrt{\frac{4 i}{m_{2}}} \psi_{L}^{2} E_{\alpha 2}^{0}-\sqrt{\frac{4 i}{m_{3}}} \widetilde{\psi}_{L}^{3} E_{-\alpha 3}^{1} \\
W_{2}^{-} & =-\sqrt{\frac{4 i}{m_{1}}} \psi_{R}^{1} E_{\alpha 1}^{-1}-\sqrt{\frac{4 i}{m_{2}}} \psi_{R}^{2} E_{\alpha 2}^{-1}+\sqrt{\frac{4 i}{m_{3}}} \widetilde{\psi}_{R}^{3} E_{-\alpha 3}^{0} \\
W_{2}^{+} & =\sqrt{\frac{4 i}{m_{3}}} \psi_{L}^{3} E_{\alpha 3}^{0}-\sqrt{\frac{4 i}{m_{1}}} \widetilde{\psi}_{L}^{1} E_{-\alpha 1}^{1}-\sqrt{\frac{4 i}{m_{2}}} \widetilde{\psi}_{L}^{2} E_{-\alpha 2}^{1}
\end{aligned}
$$

$E_{\alpha_{i}}^{n}, H_{1}^{n}, H_{2}^{n}(i=1,2,3 ; n=0, \pm 1)$ are some generators of $s l(3)^{(1)}$. The commutation relations for an affine Lie algebra in the Chevalley basis are

$$
\begin{aligned}
& {\left[H_{a}^{m}, H_{b}^{n}\right]=m C \frac{2}{\alpha_{a}^{2}} K_{a b} \delta_{m+n, 0}} \\
& {\left[H_{a}^{m}, E_{ \pm \alpha}^{n}\right]= \pm K_{\alpha a} E_{ \pm \alpha}^{m+n}} \\
& {\left[E_{\alpha}^{m}, E_{-\alpha}^{n}\right]=\sum_{a=1}^{r} l_{a}^{\alpha} H_{a}^{m+n}+\frac{2}{\alpha^{2}} m C \delta_{m+n, 0}} \\
& {\left[E_{\alpha}^{m}, E_{\beta}^{n}\right]=\varepsilon(\alpha, \beta) E_{\alpha+\beta}^{m+n} ; \quad \text { if } \alpha+\beta \text { is a root }} \\
& {\left[D, E_{\alpha}^{n}\right]=n E_{\alpha}^{n}, \quad\left[D, H_{a}^{n}\right]=n H_{a}^{n} .}
\end{aligned}
$$

\section{References}

[1] H. Blas, Higher grading conformal affine Toda theory and (generalized) sine-Gordon/massive Thirring duality, JHEP 03 (055) 03. [arXiv:hep-th/0306171].

[2] H. Blas, H. L. Carrion and M. Rojas, Non-commutative solitons and strong-weak duality, JHEP 05 (037) 03. [arXiv:hep-th/0502051].

[3] H. Blas and H. L. Carrion, Solitons, kinks and extended hadron model based on the generalized sine-Gordon theory, JHEP 07 (027) 01. [arXiv:hep-th/0610107]. 